\title{
The production of deuterated polyethylene targets
}

\author{
$N$ Kheswa $^{1, *}$, TRS Dinoko ${ }^{1,2}$, and $M$ Wiedeking $^{1}$ \\ ${ }_{1}^{1}$ Themba Laboratory for Accelerator Based Sciences, Somerset West, 7129, South Africa \\ ${ }^{2}$ National Metrology Institute of South Africa, P/B X34, Lynnwood Ridge, Pretoria, 0040, South Africa
}

\begin{abstract}
In this contribution, we describe the method used to produce deuterated polyethylene $\left(\mathrm{C}_{2} \mathrm{D}_{4}\right)$ targets. The modification of method based on polyethylene dissolution in hot xylene resulted with stable thin $\mathrm{C}_{2} \mathrm{D}_{4}$ targets.
\end{abstract}

\section{Introduction}

In experimental nuclear physics research transfer reactions (particle pickup or stripping) are a powerful tool to unravel single-particle structures in nuclear systems. Traditionally, experiments utilize transfer reactions in normal kinematics, where light beam particles (such as protons, deuterons, alphas, etc) are accelerated and impinged on heavier target atoms. Unfortunately, the inability to manufacture targets made of radioactive elements with short lifetimes (in a range of few h) limits application of classical set-up i.e. heavy target/light projectile in this type of studies.

However, with many new radioactive ion beam facilities already operational and other being currently under construction, inverse kinematic reactions are becoming increasingly important for nuclear physics experiments. Deuterated polyethylene foils, which are easy to handle are widely used in inverse kinematics measurements. Experimental requirements often seek kinematic energy losses of the beam in the target foil to be minimized, while the targets also have to be able to withstand radiation damage and melting due to the bombardment by incident heavy ions. While for many experiments, a thin target is desirable, others may require much thicker one to increase the signal strength and other deuterated materials, such as deuterated polystyrene. Deuterated polyethylene targets are favourable because they are more resilient to radiation damage and have a higher melting point. Such targets were previously prepared at other laboratories, for example by dissolving polyethylene in warm xylene [1] or using a hot presser [2]. In this work, we report the production of $\mathrm{C}_{2} \mathrm{D}_{4}$ thin foils applying modified Arnison method [1].

\section{The method development}

The natural polyethylene powder supplied by Sigma Aldrich was used for method development. The relative density at $25{ }^{\circ} \mathrm{C}$ of the polyethylene was $0.92 \mathrm{~g} / \mathrm{ml}$ with the average molecular weight $(\mathrm{Mw})$ of $\sim 4000$ and average in molecular weight was $(\mathrm{Mn}) \sim 1700$. Initially, the polyethylene powder was added to hot xylene and when dissolved it was then transferred directly onto glass slides whilst still hot. In another attempt, the dissolved hot polyethylene was allowed to cool down to room temperature before pouring onto glass slide or dipping the slide in already cooled polymer solution. It was found that both prepared solutions upon cooling separate into two layers with the crystallising polymer at the bottom layer.

In another tests, reactants, the polymer and xylene were heated at the same time. And once the solution reached the boiling point, the hotplate was switched off. The polymer solution was allowed to cool down to room temperature before pouring it onto the glass slides. This solution also formed two separate layers as previously mentioned. When hot solution was poured directly on glass substrate, the white crystalline on substrates was observed upon drying, Figure 1(a). This polymer film could not be released from the substrate even after heating the substrates on the hot plate. The homogenous $\mathrm{PE}$ in xylene solution was finally obtained when the solution was stirred continuously during the heating and the cooling; Figure 1(b) presents PE layer/film obtained from homogeneous solution.

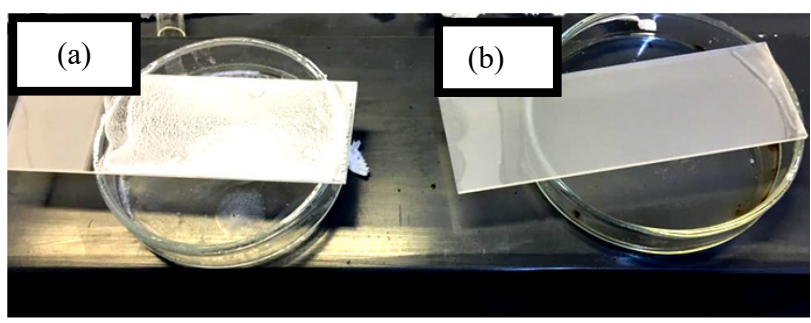

Fig. 1. Glass slides with a natural polyethylene showing the crystallisation of polymer (a) from the solution that formed two separated layers and the slide on (b) is from a homogeneous solution, no. 3 


\section{Production of deuterated polyethylene targets}

The deuterated polyethylene $\left(\mathrm{C}_{2} \mathrm{D}_{4}\right)$ material is supplied at relatively high costs compared to natural polyethylene, therefore $\mathrm{C}_{2} \mathrm{D}_{4}$ targets were reported to be produced in a controlled environment to implement cost saving measures $[1,3,4,5]$. The method reported by various authors involved dissolving polyethylene in xylene $[1,3,4,5]$. From these reports, warm, hot or boiling xylene was reported to be used and some authors reported using parting agent on substrates or just polished substrates with no parting agent. In other instances, $\mathrm{C}_{2} \mathrm{D}_{4}$ targets were reported to be covered with thin film of carbon to enhance their longevity under the beam bombardment [3]. Nevertheless, in all these papers the heat treatment of the deposited polyethylene for the polymerization and evaporation of xylene is reported as the crucial step during the production process. Therefore, we report on the modified method that produced positive results and generated strong targets that were required for nuclear physics experiment to be performed at iThemba LABS. The details of the chain configuration of the deuterated polyethylene $\left(\mathrm{C}_{2} \mathrm{D}_{4}\right)$ also supplied by Sigma Aldrich are not available on the supplier's specification sheet, however the isotopic enrichment of the polymer was $98 \%$.

The $\mathrm{C}_{2} \mathrm{D}_{4}$ powder of about $1 \mathrm{~g}$ was dissolved in $40 \mathrm{ml}$ xylene in a glass beaker. The beaker with the constituents was covered with the watch glass and was placed inside the glass petri dish in order to distribute heat evenly during heating. This was placed on top of the hot plate; see Figure 2(a). The temperature of the hot plate was increased slowly to near the boiling point of xylene $\left(\sim 145^{\circ} \mathrm{C}\right)$ and then kept in this temperature for further 5 minutes. This was then followed by the temperature decrease of $50{ }^{\circ} \mathrm{C}$ for every 30 minutes until the hot plate was switched off completely. The solution was let to cool down to room temperature. It should be noted that the polymer solution was continuously stirred during the heating and the cooling down processes. Due to this continuous stirring process, a homogeneous solution was formed in contrast to the two-layered solution which was obtained when the solution was not stirred. Based on the Demaret observation that $\mathrm{C}_{2} \mathrm{D}_{4}$ polymerizes at $70{ }^{\circ} \mathrm{C}$, at this work the polymer solution was poured onto the slides placed on the hot plate set at $75{ }^{\circ} \mathrm{C}$ for polymerisation [5], Figure 2(b). The polymer formation on a glass slide can be observed, surveying changes of the deposit from cloudy solution into transparent solid. The slides with transparent $\mathrm{C}_{2} \mathrm{D}_{4}$ were scraped on the edges and placed flat in a petri dish filled with boiling water for releasing the polymer. The polymer floated easily to the surface of the water without any parting agent used. The solution can be saved and used for several days with similar results.

\section{Discussion and results}

Solutions 1 and 2 were obtained during the early developments of the method. Both solutions obtained when PE was dissolved in hot xylene, which formed two layered solution upon cooling, with a polymer at the bottom.

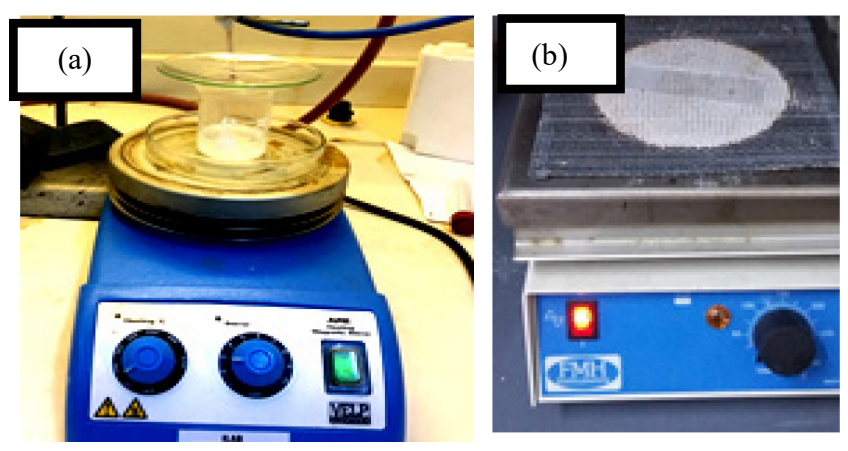

Fig. 2. The images showing (a) the heating processes used during the production of $\mathrm{C}_{2} \mathrm{D}_{4}$ targets by dissolving the powdered deuterated polyethylene in xylene and (b) the polymerization of $\mathrm{C}_{2} \mathrm{D}_{4}$ on a heated glass slide

Stirring during the heating and cooling down process of the polymer solutions yielded homogeneous solutions. Homogeneous solutions 3 to 5 presented in Figure 3 were obtained using this procedure. Solutions, 3 and 4 contain $\mathrm{PE}$ and 5 is the $\mathrm{C}_{2} \mathrm{D}_{4}$ solution. The heating of the substrates with deposited polymer for polymerization and use of boiling water for the release of polymer film from the substrate influenced production yield of strong targets which lasted for almost 5 hours of bombardment with a beam. Experiments were targets were used at involved beams of ${ }^{84} \mathrm{Kr}$ and ${ }^{132} \mathrm{Xe}$ projectiles with energies of $338 \mathrm{MeV}$ and $530 \mathrm{MeV}$ respectively.

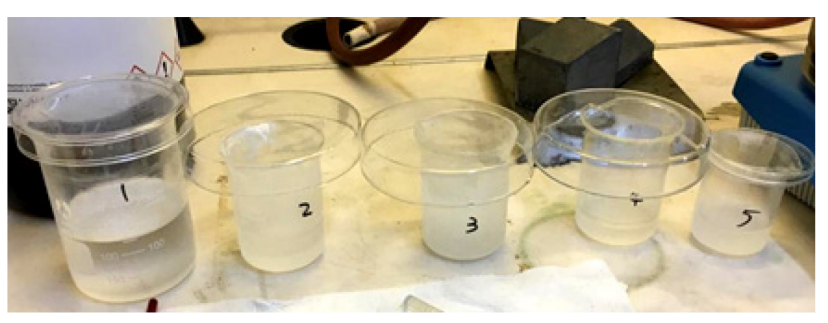

Fig. 3. Beakers containing PE (1-4) and $\mathrm{C}_{2} \mathrm{D}_{4}$ dissolved in xylene (5)

\section{Conclusion}

The method was successfully developed for the production of $\mathrm{C}_{2} \mathrm{D}_{4}$ targets. One of the crucial aspects of the method was the stirring the solution components (polyethylene and xylene) during heating needed for PE dissolving and during cooling down of the obtained solution what in turn yielded a homogeneous polymer solution. The heating of the glass substrate at the polymerisation of $\mathrm{PE}$ and $\mathrm{C}_{2} \mathrm{D}_{4}$ and using boiling water also improved the step/process of release of polymer film from the glass substrate without the application of any parting agent. Targets with thicknesses ranging from 0.1 to $3 \mathrm{mg} / \mathrm{cm}^{2}$ were produced with this method. The thickness of targets was determined by weight area measurement. The $1 \mathrm{mg} / \mathrm{cm}^{2}$ targets reported in this paper, are already used in the nuclear physics experiment conducted at iThemba LABS. 
The presented work is funded by the Department of Science and Technology of South Africa through the National Research Foundation.

\section{References}

1. G.T.J. Arnison, Nucl. Instr. And Meth. 40, 359 (1966)

2. M. Kusuhara, Nucl. Instr. And Meth. M, 83, 328 (1970)

3. G.E. Tripand, B.L. White. Rev. Sci. Instrum. 38, 435 (1967)

4. H. Fraiquin, INTDS newsletter, 21\#2, 13 (1993)

5. P. Demaret, INTDS newsletter, 19\#1, 2 (1992) 